\title{
Review: Ecology of Virtual Realities
}

\author{
Assen I. Dimitrov \\ Institute for the Study of Societies and Knowledge, Bulgarian Academy of Sciences, Bul- \\ garia, assen.dimitrov@gmail.com
}

\begin{abstract}
The book's introductory chapter justifies its overall theoretical, ecological framework, its scientific and cultural assumptions, and the range of the philosophical, interdisciplinary, and particular issues arising in the context of the ecology of virtual realities. The second chapter outlines a complementary perspective on virtual reality, disclosed by the philosophy of information, the proper categorical structures and relations to which the complex dynamics of virtual reality boils down. Chapter 3 extensively deals with the architecture and the design of virtual reality, the augmented reality, cyberspace, and the prospects for its future. Chapter 4 examines the auto/regulation of the virtual public sphere, considered as a digital ecosystem. The fifth and final chapter introduces us into the machinery underlying the phantasmagorical logic of virtual financial markets.
\end{abstract}

Keywords: Ecology of virtual realities, Philosophy of information, binary approach, symbolic representations, architecture and design of virtual reality, augmented reality, cyberspace, virtual public sphere, digital ecosystem, deliberative democracy, transubstantiation and virtualization of primordial economic realities, financial derivatives, leverage, Baudrillard simulacra, crypto-currencies

Gungov, Alexander, Alexander Lazarov, Plamen Makariev, Silvia Mineva and Olya Harizanova. Ecology of Virtual Realities. 2015. Projectoria, Sofia. 154 pages. http://www.projectoria.com. ISBN: 978-619-156-117-9, (PDF), 978-619-156-118-6 (ePub), 978-619-156-119-3 (mobi)

The attitude of mass consciousness to virtuality to date is quite polarized. On the one hand, there are those who tend to ascribe a fully-fledged ontological status to the virtual environment, if not more sustainable than that of the objective reality. This tendency of mass consciousness is also considered by the authors of the book-e.g. A. Gungov notes that one of the forms of today's virtual money, the minacoin, enjoys in the web market a higher confidence than real currencies. On the other hand, virtual reality is often underestimated and denigrated-as an illustration I would cite an eloquent comment from an anonymous forum author, who wrote that for him Internet is like a hammer: if he needs it, he uses it; otherwise, it stays in the drawer and he may not think of it for months.

Yet, it seems the most established popular illusion about virtual reality (VR) is that it is a parallel, imaginary reality. However, if we decide to look at the imaginary cyberspace as something that has acquired its own existential status, out and with no reference to the current physical and social reality, we will have to sooner or later admit that we are actually caught in a stunted or degenerate "magic" world, that of Don Quixote's battle with the windmills, or to date-in the world of the nerds ${ }^{1}$ and the trolls- that also has not escaped the attention of the authors of this original and representative survey.

Virtual reality is neither a fashionable information tool and does not end even with its highest instrumental, i.e. software applications, nor is it some imaginary parallel world. Rather, with the emergence and development of information society, it became possible in practice to delete the border between the imaginary (virtual in the classical sense) and the actual. Computer images have entered in the field of natural, physical, and sensory objectiveness and even it has become possible to cause real, physical operations only with the power of mind. There, to such a complex, controversial and even more challenging with the unknown

\footnotetext{
${ }^{1}$ People, living on a carpet, laid in front of a computer screen, unable to break away from it even under the pressure of their primary physiological needs.
} 
and the irrational about it, environment, the authors of this monograph, concise as a volume, but the more intense, synthetic and large-scale as a prospect, have set off.

\section{Ecology of Virtual Realities: What is it?}

In the book's introductory chapter, Silvia Mineva justifies its overall theoretical, ecological framework, its scientific and cultural assumptions, the range of the philosophical, interdisciplinary, and particular issues arising in the context of the ecology of virtual realities, as well as its foreseeable existential projections in the image of human future.

The outline begins with the genesis and evolution of the category: from the original treatment of E. Haeckel, through the establishment of the term in the mass consciousness as a concern for the environment and leads to today's dominant orthodox interpretationconnecting ecology only with the dynamics of nonhuman populations and the expansive one, finding in ecology a new merging point of all sciences and their integration into management. The expansive paradigm contributes to the further proliferation of the term as referring to an abundance of "ecologies" that cover and study the specifics of a huge number of natural and "artificial" (abiotic, cultural) environments.

Having originated as a branch of biology, ecology has gradually emancipated from its close biological meaning and has become an independent scientific paradigm, based on an interdisciplinary approach. Its interdisciplinarity is imposed by the complicated ecological task to explore the interdependence and interrelation of phenomena and systems of a different order as "exchange" at different levels and of different types-matter, energy, information, symbols, ideas, etc. As far as the technological revolution is now measured in the digital domain as the most spectacular and fast growing area of computer systems integrated in games and entertainment, scientific activities and communications, it is a subject of Ecology of virtual realities.

From here, Mineva heads out to the core of the ideology justifying the integrity and focus of the book-why the team of authors examines VR namely as an ecosystem, besides a digital one. There are two reasons: First, is that VR is a non-hierarchical system; Second, it represents a particular environment, an "ecosystem" that allows and encourages interaction and interdependence between humans and the machine.

To call attention to the first statement, VR cannot be considered as a homogeneous medium, subject to universal principles, rules, and laws, nor a common disciplinary framework, nor identical theoretical tools for its description and study are possible. To this should be added the emergent dynamics of VR, in which processes seem to spring from obscurity; they are wiped out by the element of the transient and the trivial. VR, as an environment, can neither be a subject to unification, nor to sustainable systematization and exhaustive mapping, not even to mention about a reliable external control. Hierarchy is absent from VR not only due to its excessively chaotic nature and even the anarchic "layout" of its major driving forces and agents, but also because here the asymmetric impacts and responses are generally the most effective ones. A healthy ecology of human civilization implies that the flexibility of the civilization shall match that of the environment to create an ongoing complex system, open ended for slow change of even basic (hard-programmed) characteristics-Mineva notes.

The authors of the following chapters subsequently develop this line of reasoning on the non-hierarchical order of the dynamics of VR by adding new conceptual structures and relations to it. Thus, VR turns ultimately to be a new quasi-physical environment where hierarchy and the deterministic connections are replaced by freedom, rationality, and meaning.

\section{Ecology of Virtual Environments Analysis in terms of Philosophy of Infor- mation}

In the second chapter, Alexander Lazarov focuses on the proper categorical structures and relations to which the complex dynamics of VR boils down: bits, binary approach, symbolic representations; the three axes of the complex space of human reasoning and action: real, imaginary, and informational. 
Lazarov outlines a new, complementary perspective on virtual reality, disclosed by the Philosophy of information (PI). Here are some of the unquestionable scientific facts and conclusions that PI identifies as foundation for its views:

- The nature of the physical world we inhabit is associated with the trinity of matter, energy, and data bits;

- There is a direct connection between material bodies, energy, and data, and each of these elements can be converted into another in the sequence matter-energy-data and vice versa. Every physical, chemical, biological, social, or other process in which there is an exchange of electrons, in fact, represents data exchange;

- The sum of all elements of IT equipment and the respective networks are the essential result of the discovery of digital components in the world.

Besides, Lazarov draws a clear demarcation between data and information, namely: each bit (multiple bits) represents data, while the information consists of bits specifically recalled from memory or purposefully retrieved data to fulfil a particular goal. In this context, there is no information without representation and more; unlike data, information is always associated with an intelligent agent, regardless of its level of intelligence.

Essentially, bits are always written as "0" or "1", respectively symbolizing "no" or "yes". Bits are the result of a binary approach, especially typical of human perception, which always seeks to position each "any new" information in the interval between two opposite and contrasting ends, for example, near-far, hot-cold, happy-unhappy, and so on. The synthesis of the binary approach and symbolic representation is the heart of IP, which in its turn is the groundwork for contemporary computer technology and its virtual informational, existential, and social projections.

Thus, after all, virtual processes are the sum of capacities and actions for:

- Identifying and extracting (in some cases, deduction or decompression) of the component of bits from the trinity matter-energy-data using natural and/or artificial sensors or detectors;

- Transducing their signals to the processor, including the brain;

- Transforming them into signs and codes;

- Saving-recording and storing of newly received signs and codes on another/new carrier type of database.

Or, to cut it short, first material dynamics is converted into binary data, i.e. each continual process is converted into a discrete one, which, moreover, can occupy only one of two possible polar states; then data are stored into symbolic representations that are processed under the rules of various algorithmic codes.

Next, the author puts forward a typology of the basic capacities of intelligence with regard to IP. To this end, he considers the complex plane proposed by Descartes and the following analysis of it: The complex plane unites rational numbers (along the $X$ axis) and imaginary numbers (along the $\mathrm{Y}$-axis). That is, each point of this plane has a real component extracted or taken from the physical world and an imaginary one, expressing the image constructed by human imagination. Lazarov, however, supplements a third axis $Z$, the combination of sequestration capacity, memory, and information processing by intelligent systems. Thus, each point of this space will be characterized not only by the unity of reality and imagination, but also by the capacity for perception, evaluation, reasoning, and creativity of the specific system while maintaining full freedom of choice of the individual point of view and perspective.

\section{Genesis and Development of Virtual Reality Computer Systems}

In chapter 3, Olya Harizanova extensively deals with the architecture and the design of VR, the augmented reality, and cyberspace and the prospects for its future. From a technical perspective, VR is a term used to describe the three-dimensional (3D) computer-generated envi- 
ronment within which one can interact or explore. In point of fact the users (human beings) become part of the generated virtual world by "immersing themselves" in the simulated environment and at the same time being able to handle different objects and perform a number of actions. For this purpose, they must be equipped with special devices, (e.g. goggles through which to visualize 3D images (head-mounted display; HMD), special gloves with sensors etc.

The communication interface comprises of: natural interface for navigation in 3D space (viewing, moving, or flying in the surrounding simulation); stereoscopic view, enhancing the sense of depth and space; normal scale appropriate to human size; and opportunity for realistic interaction with virtual objects using special equipment to manipulate and manage the simulated system. Ultimately, VR is the product of the application of computer-based technologies to create interactive three-dimensional effects in which objects and sources of information have their own spatial "presence", Harizanova points out.

Here I can only mention the author's detailed outline of the evolution of VR technology including its basic stages and several complementing typologies. The author discusses several types of VR: fragmented, simulated, augmented, hybrid, and combined. The reader should attentively consider Harizanova's analysis of augmented reality (AR) in particular. In short, $A R$ is a system of tools allowing the user to view one or more virtual objects in the middle of the surrounding natural reality by overlaying computer graphics.

As to the present and oncoming developments in the realm of VR, the author makes a clear and vivid comparison; for example, let us assume that someone wants to read certain information. The conditional instructions when working with a traditional computer or mobile device would be as follows: "you type on the keyboard" or "select download" or "touch and choose". The result is often a pop-up window on the monitor/screen in which the user is prompted to make a series of such actions.

How does all of this happen in VR? For the same activities (reading data), the conditional instructions to the user would be, quite simply as follows: "slide these windows from your screen in the space in front of you; use your eyes to switch the lights on, to open doors or surf the internet."

The author's handling of the agents, communicating, and interacting in VR, especially the three-dimensional or two-dimensional images of the user in the virtual environment, the avatars, will certainly also capture the readers' attention.

\section{The Virtual Public Sphere as a Digital Ecosystem}

In chapter 4, Plamen Makariev examines the auto/regulation of the virtual public sphere, considered as a digital ecosystem. He starts with a brief presentation of the generic feature of the public sphere as a communication network whose main function is to de-/legitimize norms, policies, and practices concerning socially significant issues. As to its differences of kind, the author considers two most important ones.

The first one is unforced communication that occurs in the network. Its effect is produced through relying on persuasion rather than on the threat of sanctions. The public sphere must be independent not only of governmental, but also of economic coercion. Hence the public sphere occupies a "space" located between the institutions of power, economic life and the private life of individuals and groups. It mainly operates in the realm of civil society, but in a broader sense, includes political parties, which are not involved in governance at the time, and also with the participation of the institutions of power, as far as it is limited to the dimension of legitimizing norms, policies, and practices. The basic form of realization of the public sphere is the formation of public opinion and its impact on social life.

The other difference of kind of the public sphere as a communication network, with de/legitimizing functions, is its openness-hence the very term "public". In regard to the openness of public communication, its virtualization provides new opportunities for thematising problems, which for one reason or another are being ignored or played down by the mainstream media. A poignant and timely saying uttered in virtual space can reach a multimillion public instantly, to be picked up by multiple virtual "voices" and have a greater impact on 
public opinion than any systematic manipulation of the latter by a media empire, the author notes.

It is a fact that mass brainwashing leads to depersonalization of participants in public life and makes them to some extent subject to the control of the institutions, corporations and so on. On the other hand, however, mass media in democratic countries in general, contribute to the maintenance of a social homeostasis, while widening opportunities to individuals and small groups to significantly affect public sphere, increases the risk of social and political turmoil. The anonymity of participation in Internet debates and the unlimited freedom in taking a stance, introduces an element of anarchy in public communication, weakening the legitimacy of the rules and norms (policies, practices) that receive public support and what is worse, may reinforce extremist ideologies and even political practices.

Besides, there is richness and diversity that is unmatched with the content of the publications of professional journalists in the traditional media. This creativity could contribute to finding solutions to complex social and political problems, yet unfortunately, in most cases it is "drowned" in streams of destructive participation in the network.

Next, Makariev focuses on the issue of the optimization of digital publicity in its capacity of a moral social regulator; of the overcoming of the subjective and manipulative distortions in it. This can be done, he suggests, through a symbiosis of the mechanisms of its spontaneous self-regulation and the compliance with the principles of social deliberative democracy.

Self-regulation of legitimating public communication could probably be done in various ways, but one of them has to be the "deriving" of public-deliberative arguments from various forms of persuasive communication and the prioritization of their legitimizing or delegitimizing effect in regard to the discussed rules/norms or policies, or practices, Makariev concludes.

\section{From Virtuality of Gold Coins to Virtual Currencies Backed by Gold}

In the fifth, final chapter, Alexander Gungov introduces us into the objective mechanisms underlying the phantasmagorical logic of virtual financial markets. The chapter can be divided into two basic parts: the first, starting from Marx's complex analysis (based on the method of ascending from the abstract to the concrete) of money and tracing out the most recent developments, explains how money departed from the actual economic realities to an imaginary, phantasmagorical reality of their own. The second part considers exactly the opposite problem: how to return money back into actual societal dynamical space and whether VR can somehow lend a hand to this critically needed transformation of theirs.

Gungov establishes that derivation is the basic and most effective procedure of the transubstantiation and virtualization of primordial economic realities. The immanent/introvert ideal substance of value and the displayed/extroverted form of value, i.e. its exchange value, are for Marx in a relation of original to derivative. This operation is called conversion (Verwandlung), which is not simply transformation but a radical change comparable to the transubstantiation of bread and wine into the flesh and blood of Christ. The first and fundamental conversion is the passage of the immanent substance of value onto externalised exchange value, measurable in money. Two other significant "transubstantiations" are the conversion of surplus labour and surplus value into profit and of surplus labour into interest. Here the derivative ideal/virtual, which for Marx is also imaginary, enchants the participants in the monetary commodity market relationship, making them oblivious to the primary ideal nature of socially necessary labour as a value producing and determining condition.

Next Gungov proceeds to completely new forms of financial derivation that occurred stormily in post Marxian and especially in the current risky neoliberal market environment. Here second, third, fifth and perhaps even higher order derivatives emerge. If a bank delivers on the stock exchange part of its mortgage loans and the forecast profit from these loans for a period of time, the bank will have created a derivative. If the derivative is purchased by an insurance company and resold to a pension fund, which in turn releases it for sale to another financial market, two more derivatives are created. The essential difference between the first derivative, the one dependent on future profits on any mortgages on the one hand, and the 
second and third derivatives on the other, is that the former follows and relies on the fate of a simple financial instrument, while the latter two depend on a derivative instrument, i.e. on a derivative and are therefore derivatives of a derivative.

Derivatives are increasingly cut off from the life-bearing source-surplus labour-and lead an increasingly independent and self-sufficient being in an unconditional way. They are not obliged to refer to a reality outside of them and de facto they do not. They produce their own reality and are similar in that to the code of Baudrillard simulacra, Gungov observes.

Things extend not only to the economic sphere but continue to the broader social reality and expand even in the world of values. Here a completely different conversion/transubstantiation of money occurs, which consists in turning money from a medium into a motive. Money has replaced God, and God had previously replaced human relations; therefore, the entire operation performed by money becomes double substitution. This metamorphosis can be seen as a virtualization of virtuality of second order, which is commensurate with the conversion of second order as in financial derivatives.

The author continues with the abstract substance of electronic money. Things nowadays acquire not just a curious or mysterious, but rather a most critical character. As money more and more departs from its hidden economic essence, surplus labour, it acquires even more phantasmagorical or rather hyper-real essence. Today money is not created by national governments and central banks, although both governments and central banks could regulate money creation. Networks of commercial banks create money by extending loans with the help of a financial mechanism known as leverage. Leverage has proved quite a simple technique for multiplication of cash flows, in which the bank can lend against every received monetary unit loans, multiplied by the leverage of the bank.

So modern economics and financial science come up to the problem of "the recreation of money". An ecosystemic approach offers several solutions. Some authors believe that the state should undertake the issuance of money through taxation, returning it into society without interest. Another approach would be to introduce into economy additional currencies that circulate along with the official ones. Gungov himself emphasizes on the possible contribution of electronic money or crypto-currencies.

What remains beyond doubt is the much more stable and predictable nature of goldbacked crypto-currencies. Thus the most virtual currency invented to-date acquires the centuries-long proven and secure virtuality of gold as a measure of value, provocatively swimming against the tide of permanent insecurity and emergency, the author sums up.

\section{Final Notes}

Let me now share my overall impression of having the privilege of being among the monograph's first readers. The text is particularly concentrated. The ideas are presented clearly and accurately. The new information is systematically arranged. The compositional logic is sound: starting with the general ideological framework, passing through the ontology of $V R$, continuing with the architecture and design of VR, with digital public communication, and ending up with the transformations that logic and rationality have experienced in the context of VR.

The monograph itself is ecology of complementing and co-evolving ideologies and theoretical frameworks. This lends it an envious sustainability and semantic variety the reader should test and appreciate oneself. Did the authors manage to encounter the challenges they have posed to themselves? I would end up with a summary of two basic suggestions they, in my modest and limited judgement, achieve to accomplish into the reader's mind.

First, the authors have extended the standard imaginary (mental) dimension of VR. Thus VR incorporates new modalities, besides fantasy, as mediums of contact with the imaginary world. So the imaginary becomes an object not only to fantasy, but also to all of the five basic senses. On the other hand, fantasy itself gets direct access and even volitional control over the actual physical objectiveness. It really is already enough to wish something so that it happens, as is the case, for example, in some of today's cutting-edge applications of VR in medicine. 
Second, and even more importantly: the monograph succeeds in the explication of VR as a new technogenic cultural space-the interface of communication of fantasy with technology. From this viewpoint, VR should by no means be mixed up any longer with the imaginary human world. VR is not a mental, but a symbolic medium. It is a complex semiotic technological digital environment. A symbolic, or to put it more generally, a semiotic environment that strongly tends to largely replace its actual physical detonates. A semiotic complex technological digital environment, which shows a tendency not only to compete with the immediate physical and social environment, but to assimilate, to control, and to dominate it.

It's too early to judge optimistically or not as to the perspectives and horizons of this new reality, as it is definitely clear that they are among the few sustainable landmarks into the landscape of our blurred and critical future.

\section{The Authors of the Monograph}

Alexander Gungov is Professor of Logic and Philosophy Taught in English at the Department of Logic, Ethics, and Aesthetics, University of Sofia "St. Kliment Ohridski". His research is in the field of philosophical dimensions of logic as well as in applications of logic to medicine and to the critique of manipulation in the public discourse. He is the Director of the M.A. and Ph.D. Program in Philosophy Taught in English and Editor of Sofia Philosophical Review. He has given presentations in numerous European and American Universities.

Alexander Lazarov is a professional camera operator. He is a doctoral student at University of Sofia "St. Kliment Ohridski", in the doctoral program in Philosophy Taught in English at the Philosophy Faculty. His dissertation research project is entitled "The Digital World-a construction and reality". Scientific advisor: Prof. Alexander Gungov.

Prof. Plamen Makariev, PhD, member of the Faculty of Philosophy at Sofia University "St. Kliment Ohridski". His teaching and research work is in the fields of Philosophy of Politics, Philosophy of Education and Philosophy of Culture. From 2000 until 2011 he had been a director of "Virtual Culture" master's degree at Sofia University.

Prof. Silvia Mineva, PhD, teaches ethics at University of Sofia "St. Kliment Ohridski". She has been a head of "Logic, ethics and aesthetics" department since 2008. From 2009 Prof. Mineva is an editor in chief of "Virtual culture" e-magazine; and from 2008 until 2014-a president of "Association Of Univesity Teachers Of Philosophy In Bulgaria". He is a researcher and author of monographs, papers and articles in the fields of moral philosophy, professional and applied ethics.

Prof. Olya Harizanova, $\mathrm{PhD}$, teaches academic courses in the field of the Information Retrieval Systems. A head of "Library and Information Studies and Cultural Policy" department of the Faculty of Philosophy at University of Sofia "St. Kliment Ohridski". She is Director of a M.A. program "Management of electronic content"; editorial board member of "Library" magazine; president of the "Bulgarian Institute for Development" association. She is also a researcher and author of scientific and popular publications on sustainable development of the information society.

\section{References}

Gungov, Alexander, Alexander Lazarov, Plamen Makariev, Silvia Mineva and Olya Harizanova Ecology of Virtual Realities. 2015. Projectoria, Sofia. http://projectoria.com. ISBN: 978-619-156-117-9 (PDF). 978-619-156-118-6 (ePub), 978-619-156-119-3 (mobi)

\section{About the Author}

\section{Assen I. Dimitrov}

$\mathrm{PhD}$, Associate Professor of Philosophy at the Department of ontological and epistemological investigations, Institute for the study of societies and knowledge, Bulgarian academy of sciences.

Field of research interests: complexity and intelligence, order and control in complex hierarchical (information, bio- and social) systems' dynamics.

Books: Complexity and meaning, or about intelligence. 2004. V. Turnovo: Faber; Nonalgorithmic order. 2010. V. Turnovo: Faber; Mind and control. 2014. V. Turnovo: Faber; Author of over 50 papers, 
including: Virtual Information Systems. 1998. Integrative Physiological and Behavioral Science 33 (1): 41-48; Packages: The Atoms of Communication. 2007. tripleC-Cognition, Communication, Cooperation: Open Access Journal for a Global Sustainable Information Society 5(2): 82-86. Accessed November 4, 2014. http://www.triple-c.at/index.php/tripleC/article/view/6.

Fellow of Alexander S. Onassis foundation (2001). 